BNL-108380-2015-JA

\title{
New characterization techniques for LSST sensors
}

\section{A. Nomerotski}

Brookhaven National Laboratory,

Upton NY 11973, U.S.A.

E-mail: anomerotski@bnl.gov

ABSTRACT: Fully depleted, thick CCDs with extended infra-red response have become the sensor of choice for modern sky surveys. However, the charge transport effects in the silicon and associated astrometric distortions could make mapping between the sky coordinates and sensor coordinates non-trivial, and limit the ultimate precision achievable with these sensors. Two new characterization techniques for the CCDs, which both could probe these issues, are discussed: x-ray flat fielding and imaging of pinhole arrays.

KEYWORDs: Photon detectors for UV, visible and IR photons (solid-state) (PIN diodes, APDs, Si-PMTs, G-APDs, CCDs, EBCCDs, EMCCDs etc); Charge transport and multiplication in solid media; Image processing 


\section{Contents}

1 Characterization of LSST sensors 1

2 X-ray flat fielding 1

2.1 Footprints of $\mathrm{x}$-rays and shape fitting 2

2.2 Edge roll-off and midline distortion effects in x-rays 5

$\begin{array}{lll}2.3 & \text { Dependence on the intra-pixel hit position } & 7\end{array}$

3 Characterization of CCDs using a grid of pinholes $\quad 8$

4 Conclusions $\quad 10$

\section{Characterization of LSST sensors}

The LSST Camera focal plane will consist of 21 Science Rafts and 4 Corner Rafts. The Science Rafts will have 9 CCDs in a $3 \times 3$ sensor arrangement and will be controlled and read out with three boards of custom electronics located behind the CCDs and connected to them with short flexible cables. Each CCD will have about 16 million $10 \times 10 \mu \mathrm{m}^{2}$ pixels and will be $100 \mu \mathrm{m}$ thick. The CCD sensor is subdivided in to 16 sections, 8 in the top half and 8 in the bottom half, for fast readout. For more detail on the LSST CCDs, see the reference [1].

Sensors will undergo careful acceptance tests before integration into the rafts and are expected to meet stringent LSST specifications. Additional techniques have been under development, which would be sensitive to astrometric biases and pixel size variations, an important topic of this workshop, and other subtle sensor anomalies, which may affect the science. Two of these techniques: $\mathrm{x}$-ray flat fielding and illumination through arrays of pinholes are discussed below. All measurements presented in this manuscript are based on the recent BNL data.

\section{X-ray flat fielding}

Iron-55 x-rays are widely used for the characterization of CCDs. The x-rays penetrate into the silicon an average depth of 30 microns to produce compact, submicron size clouds of about 1600 electrons (for $\mathrm{K} \alpha$ line at $-100^{\circ} \mathrm{C}$ ). After conversion the electron cloud drifts in depleted silicon towards the $\mathrm{CCD}$ gates, diffusing in the process. $\mathrm{X}$-rays from two monochromatic lines, $\mathrm{K} \alpha$ and $\mathrm{K} \beta$ correspondingly at 5.9 and $6.5 \mathrm{keV}$, are routinely used for gain calibration [2] and could be also used for diffusion measurements [3]. The registered hit shape is intrinsically symmetric so any deviation from the circular shape could be used to probe the lateral electric fields in the fully depleted CCD, for example, near the sensor edges or due to the tree-ring effect.

The goal of this study was to observe these distortions and hence validate this technique as a possible probe of astrometric biases in the CCDs. It is worth mentioning that since the $\mathrm{x}$-rays 
penetrate the silicon a considerable distance they are not sensitive to the surface. Therefore, one can hope to extract genuine astrometric bias due to the lateral electric field (or pixel size variation) and to decouple it from the photometric contribution, which is mostly due to the quantum efficiency variation on the surface. It is straightforward to accumulate large statistics of x-rays, in the limit probing each pixel many times, so in analogy to the traditional flat fielding of sensors (illumination with a uniform flux of light) we call this technique the $x$-ray flat fielding.

\subsection{Footprints of $x$-rays and shape fitting}

A typical x-ray exposure is shown in figure 1 together with found x-ray "footprints". Sometimes $\mathrm{x}$-ray hits are blended when more than one x-ray hit is included in to a single footprint. These cases are easily recognized by double energy deposition. Note that typically a footprint has only a handful of pixels with significant signal. The reason is that the LSST pixel size is $10 \times 10 \mu \mathrm{m}^{2}$ while the typical spread due to the diffusion is $3-4 \mu \mathrm{m}$. The small number of pixels in the footprint causes a problem of undersampled PSF (point spread function) and results, for certain footprint configurations, in biases in the fit parameters as discussed in detail in section 2.3.

LSST Data Management software [4] has been used for the x-ray finding and background subtraction with a typical requirement of two adjacent pixels above a threshold, which was chosen to be 5 times greater than the pixel noise. After this the footprint was "grown" by two pixels in all directions from the pixels above the threshold to include neighboring pixels and to allow for better baseline determination.

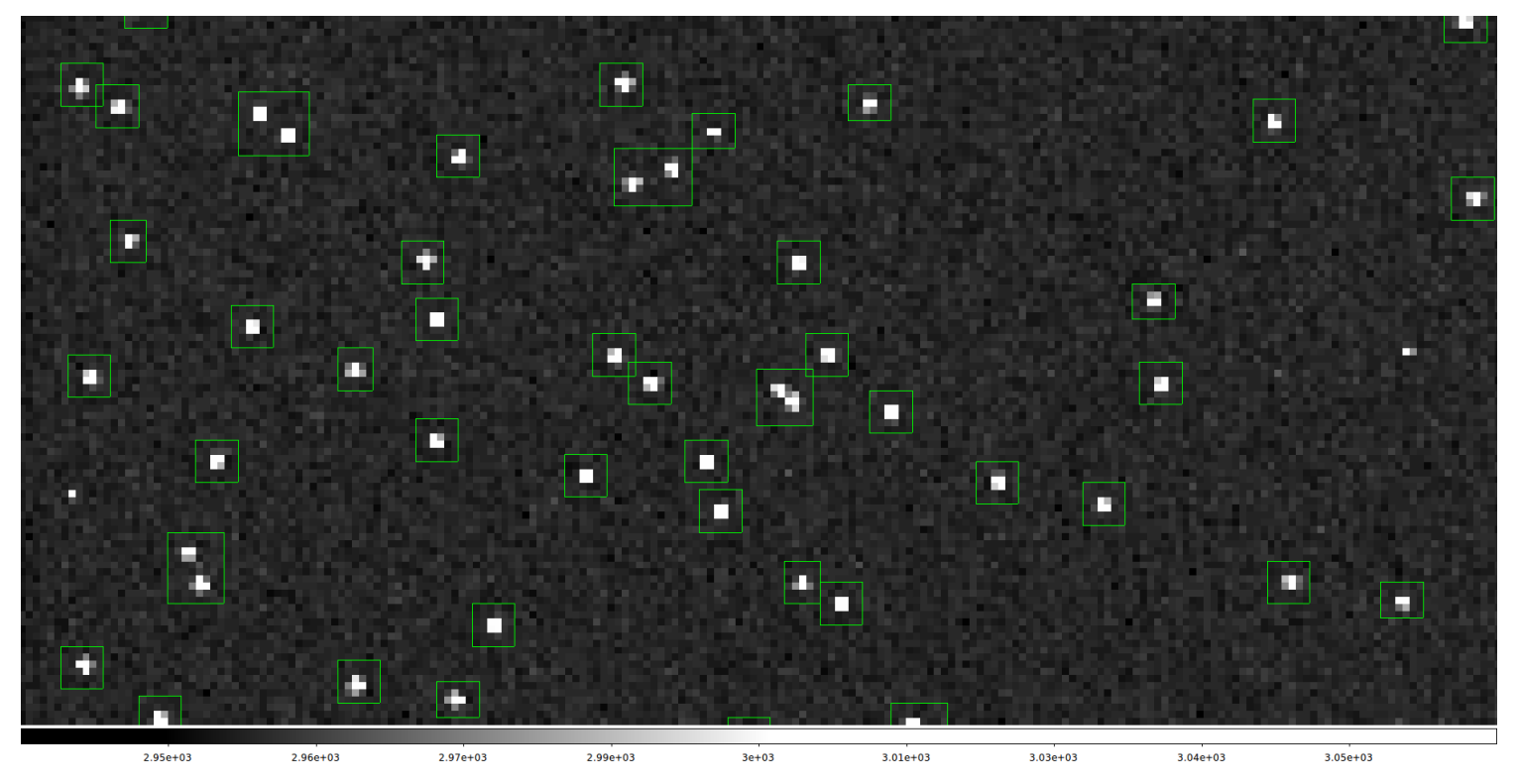

Figure 1. Typical x-ray exposure with found footprints.

The shape of each x-ray hit was determined by a 2D Gaussian fit with six free parameters: total flux, two centroid coordinates $x_{0}$ and $y_{0}$, sigma and two shears $g_{1}$ and $g_{2}$; adopting the algorithm and notations used for a weak lensing analysis in [5]. The algorithm has the capability to subdivide pixels into multiple bins, evaluating the fit function in the centre of each bin. This effectively integrates the function over the pixel if the number of bins is large enough. In our case $16 \times 16$ 
bins per single pixel have been used. This approach removes some of the undersampling bias but one needs to be careful anyway since at least six pixel values measured with enough precision are needed to extract the six parameters of the fit function, see section 2.3 for more discussion. It is worth mentioning also that fitting of stars and galaxies in LSST will not be affected since the overall PSF will be larger due to additional contributions from optics and atmosphere.

Ten million footprints have been reconstructed for the 16 million pixels of a sensor with about 7 million footprints used for the subsequent analysis after fit failures and blended hits have been removed and good fit errors selected. Figure 2 shows the $(x, y)$ occupancy map for sensor \#112-04. This preproduction sensor has a know charge transfer efficiency (CTE) issue in one of the sections, which is apparent in the map. The $\mathrm{x}$-ray source was positioned above the sensor so the occupancy distribution has a bell shape caused by the geometry.

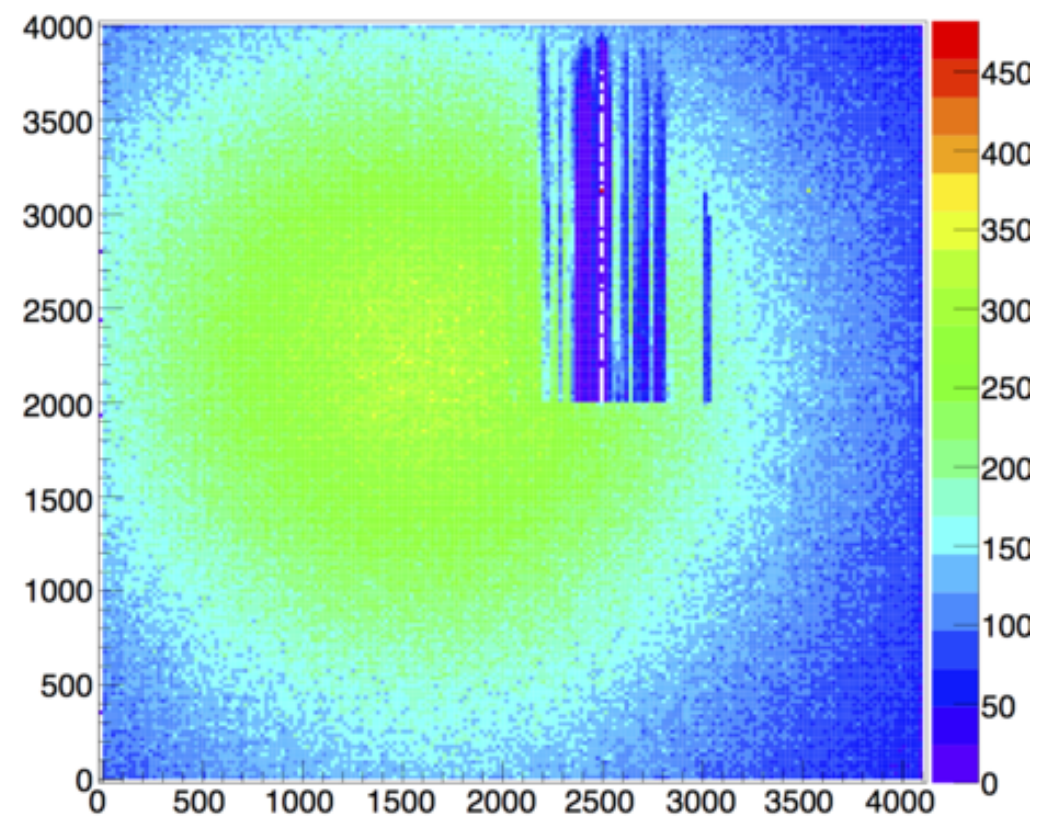

Figure 2. Occupancy map for the sensor 112-04.

Figure 3 shows distribution of measured flux, in electrons, for reconstructed ${ }^{55} \mathrm{Fe}$ x-rays using the above procedure. The data from all $16 \mathrm{CCD}$ amplifiers was gain corrected and background subtracted resulting in good separation of $\mathrm{K} \alpha$ and $\mathrm{K} \beta$ peaks, and providing excellent calibration.

Distribution of the PSF size (sigma, in pixels) shown in figure 4 is skewed due to varying conversion depth of the x-rays. Some of them convert near the entrance window so the corresponding photoelectrons drift and diffuse the full sensor thickness, $100 \mu \mathrm{m}$, before reaching the gates, while some of them convert near the gates with minimal diffusion. The distribution therefore is a convolution of the exponentially falling probability to convert at certain distance from the entrance window with the diffusion corresponding to the distance from the conversion point to the gates. This information can be used for the diffusion measurement in CCDs as described in [3]. 


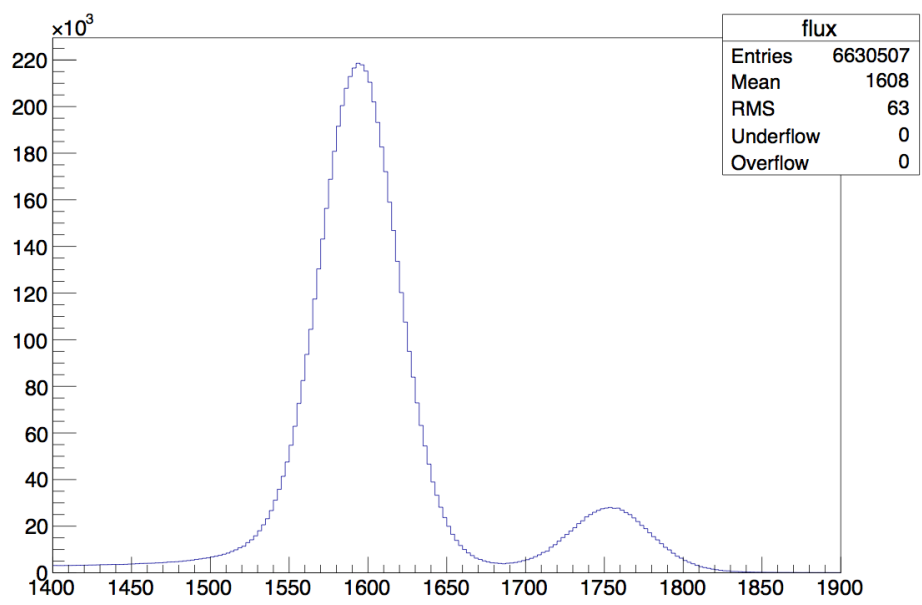

Figure 3. Distribution of measured flux, in electrons, for reconstructed ${ }^{55} \mathrm{Fe} x$-rays.

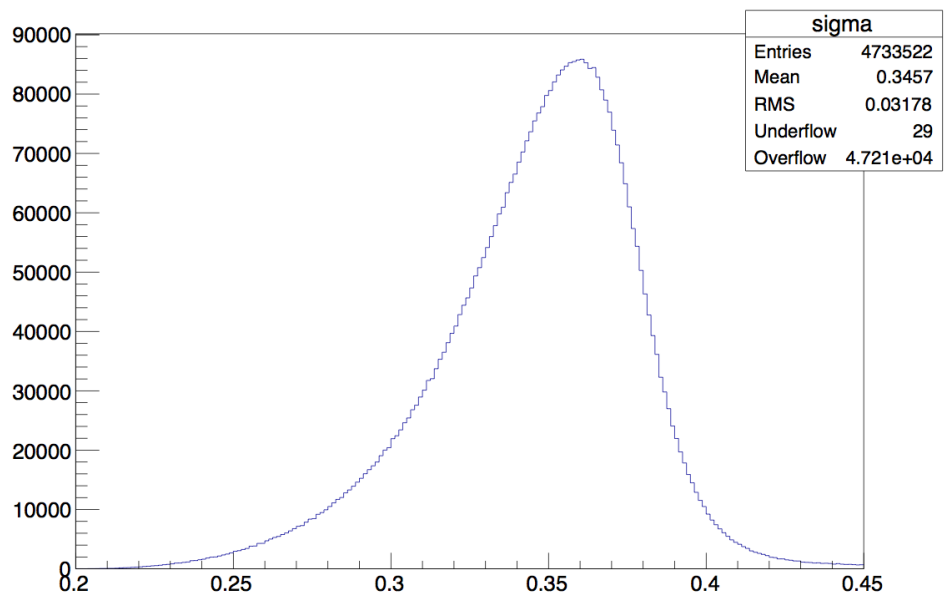

Figure 4. Distribution of the PSF size (sigma, in pixels) in the fit.

The fit parameters responsible for distortion from the circular shape are two components of the shear, $g_{1}$ and $g_{2}$. The weak lensing definition of $g_{1}$ and $g_{2}$ has been used:

$$
g_{1}=\frac{a-b}{a+b} ; \quad \tan (2 \theta)=g_{2} / g_{1}
$$

In the above, $a$ and $b$ are the major and minor axes of the ellipse and angle $\theta$ defines the ellipse orientation. The $g_{1}$ shear component is responsible for elongation along the $x$ and $y$ axes while the $g_{2}$ component is responsible for the elongation along the \pm 45 degree directions. Figure 5 shows the two-dimensional distribution of $g_{1}$ and $g_{2}$. The distribution is peaked at zero as expected corresponding to a symmetric shape. The distribution width is determined mostly by the fit uncertainties. 


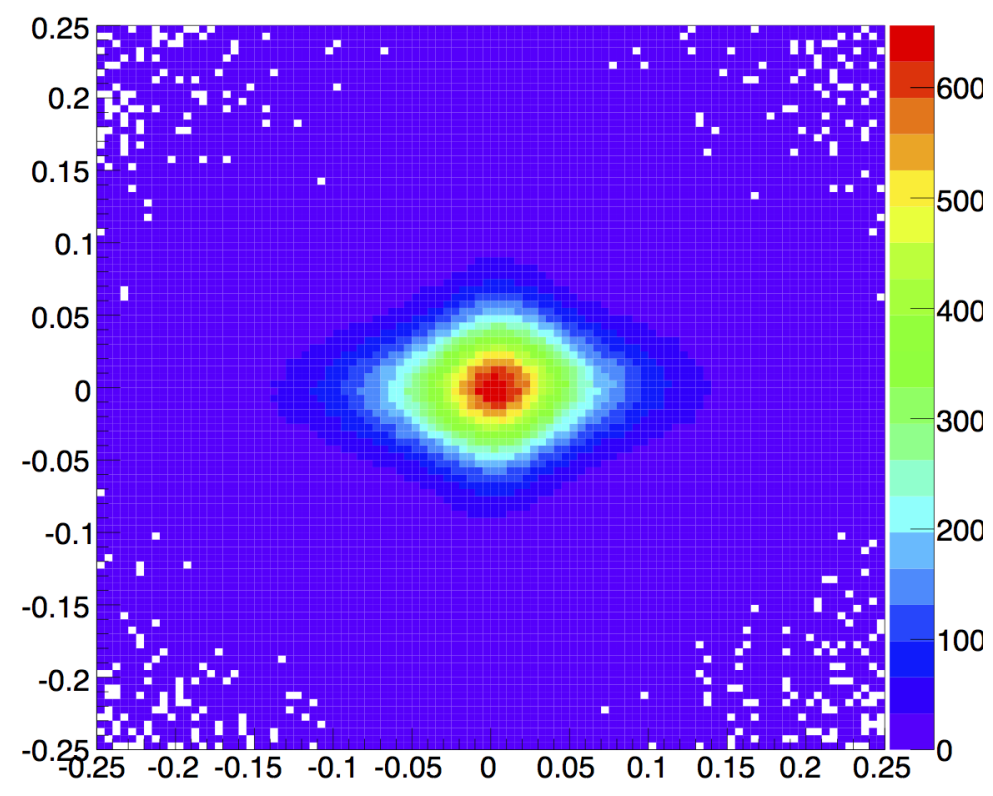

Figure 5. Two-dimensional distribution of $g_{1}$ (horizontal axis) and $g_{2}$ (vertical axis) components of shear.

\subsection{Edge roll-off and midline distortion effects in $x$-rays}

One of the most prominent manifestations of the lateral field effects in thick, fully depleted CCDs is at the sensor edge. The guard-ring, which runs along the outer perimeter of the sensor plays an important role in protecting the sensitive region from parasitic surface currents but at the same time distorts the regular structure of the field lines, causing photoelectrons to deflect out of the sensitive volume towards the guardring. This causes elongation of the reconstructed shape towards the sensor edge.

This effect has been observed in the $\mathrm{x}$-ray flat fields as illustrated in figure 6 . The figure shows the shear $g_{1}$ near the sensor edge for $x$ (along the row direction) and $y$ (along the column direction) coordinates near the corresponding edges. The shear was averaged over all columns for a particular row for the left graph and similarly over all rows for a particular column for the right graph. Error bars of the points represent RMS of the corresponding distributions. About 10 edge pixels are affected both in row and column directions, in agreement with previous measurements of the edge roll-off effect in LSST sensors using different techniques [6]. Negative values of $g_{1}$ in the left graph correspond to elongation along the x-axis, while the positive values of $g_{1}$ near the edge in the right graph correspond to elongation along the y-axis, all as expected. The $g_{2}$ shear does not deviate from zero near the edge, confirming that there are no 45 degree components in the ellipse orientation.

Another important effect of pixel size variation occurs near the anti-bloom implant between the top and bottom halves of the sensor. The p-type implant changes the configuration of electric field in the vicinity of the midline. This could also be aggravated by the real pixel size change due to the modified pixel geometry in this area to accommodate the implant. This effect causes "wobbling" 

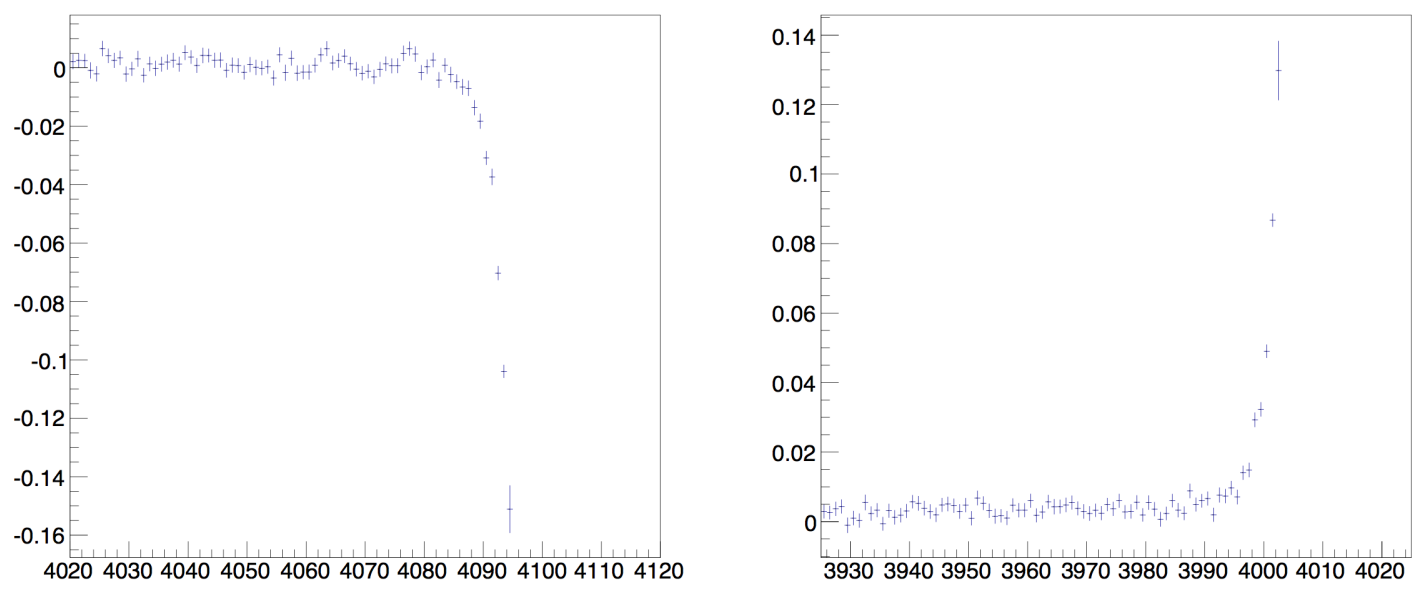

Figure 6. Left graph: shear $g_{1}$ near the sensor edge for $x$ coordinate (along the row direction). Right graph: shear $g_{1}$ near the sensor edge for $y$ coordinate (along the column direction).

of ellipticity near the pixel \#2002, as demonstrated in figure 7. The shape first becomes elongated along the row direction, then along the column direction and then again along the row direction.

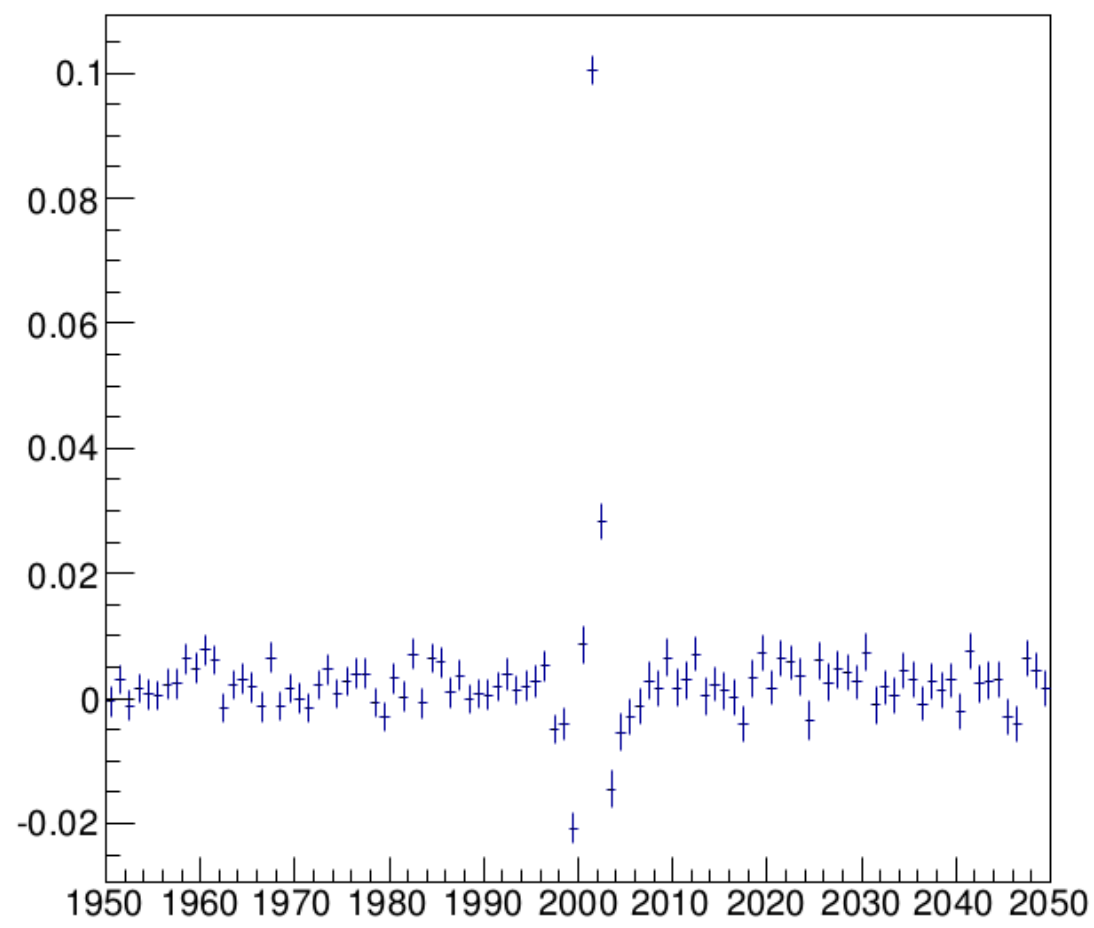

Figure 7. Shear $g_{1}$ near the sensor midline.

The edge and midline effects were also observed in the sigma (PSF size) distribution. As far as we know this was the first detection of these effects using $\mathrm{X}$-rays. 


\subsection{Dependence on the intra-pixel hit position}

$\mathrm{X}$-ray centroids can be reconstructed with good precision, about 0.1 micron, which is considerably smaller than the pixel size, 10 micron. This information can be used to map the reconstructed fit parameters as function of the hit position within pixel. For example, the fraction of the total charge collected in the central pixel as a function of the hit position is shown in figure 8 . If the conversion happens exactly in the pixel center then about $70 \%$ of the charge is registered in the central pixel with the rest distributed among the immediate neighbors. If the conversion happens on the border between two pixels at the same distance to the closest pixel corners then these two pixels collect $40 \%$ of the charge each. Lastly, when an x-ray converts in the very pixel corner the charge is split equally between all four adjacent pixels, each receiving $25 \%$.

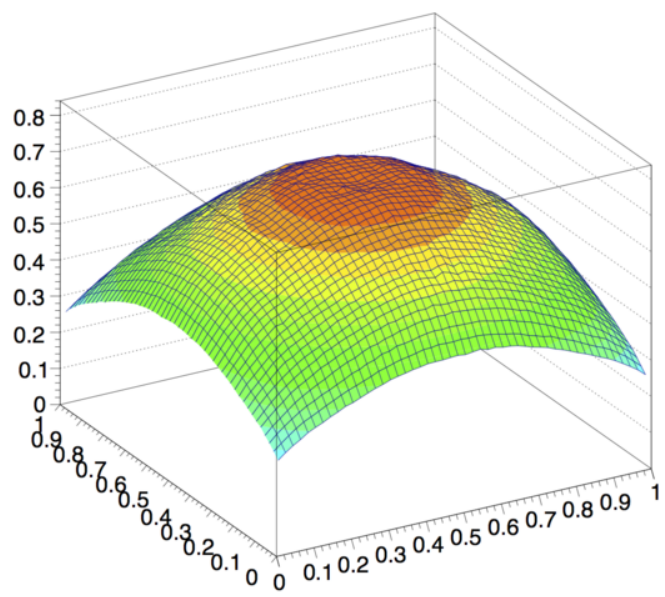

Figure 8. Fraction of the total charge collected in the central pixel as a function of the hit position.

Shear $g_{1}$ and $g_{2}$ as a function of the hit position are shown correspondingly in the left and right graphs of figure 9 . One can see a considerable bias in $g_{1}$, up to $5 \%$, in the distribution caused by small number of pixels with significant charge deposition when the hit occurs near the pixel boundary as the charge is mostly shared between two pixels. In this case the reconstructed shape is elongated either along the columns (negative values of $g_{1}$ ) or along the rows (positive values of $\left.g_{1}\right)$. The distribution of $g_{2}$ also has a bias when an x-ray hits the pixel off-center such that the charge is mostly shared between three pixels arranged in a configuration angled at 90 degrees. In this case the reconstructed shape tends to have bias along the 45 degree orientation. Note that the $g_{2}$ bias is considerably smaller than the $g_{1}$ bias.

It should be stressed that the shear averaged over the whole pixel is zero to a good precision. This means that, despite of the above bias, the reconstructed shear can be used to study the lateral field effects as described above in section 2.2 since in this case the data in each point was averaged over many thousands of hits and pixels. 

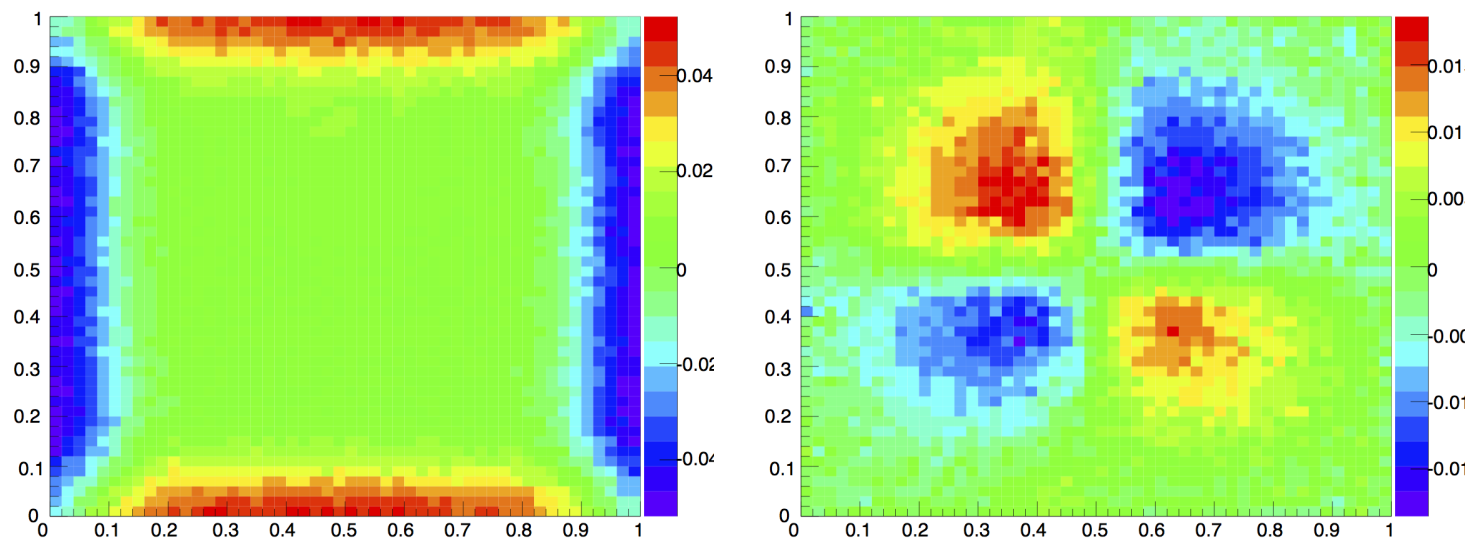

Figure 9. Shear $g_{1}$ (left graph) and $g_{2}$ (right graph) as a function of the hit position.

\section{Characterization of CCDs using a grid of pinholes}

Another technique used to probe the lateral field effects employed a regular grid of pinholes etched in $150 \mathrm{~nm}$ thick chromium deposited on silica [7]. In total, the plate had 46,656 pinholes with $3.6 \mu \mathrm{m}$ diameter and $200 \mu \mathrm{m}$ spacing. Figure 10 shows an SEM photograph of one hole.

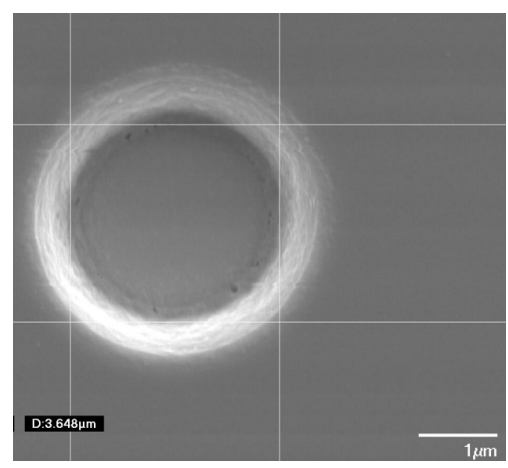

Figure 10. SEM photograph of one hole.

The plate was installed in front of the CCD using simple projecting optics with $f / 1.2$ and small demagnification factor of 1.2. Figure 11 shows a raw ds9 [8] image of the pinholes.

The pinhole images were processed using the same procedure as for the ${ }^{55} \mathrm{Fe} x$-rays described in the previous section. Compared to the x-rays the pinhole images had a larger PSF (sigma) of 6-7 $\mu \mathrm{m}$ so the undersamplig problem and corresponding biases in the shape reconstruction were not visible. Figure 12 shows an $(x, y)$ map of the reconstructed PSF size (in pixels) for a sensor segment. One can see a region of uniform PSF and also some peripheral distortions in the bottom right corner due to the optics. A uniform region was selected for further analysis to determine distances between the pinholes.

Figure 13 shows the distribution of distances (in pixels) between the neighboring holes along the row (left graph) and along the column (right graph) directions. The distributions are narrow 


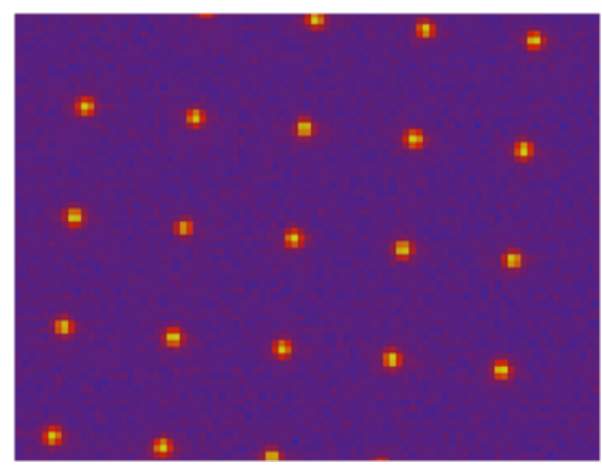

Figure 11. Raw image of the pinholes. The distance between pinholes is about 168 micron.

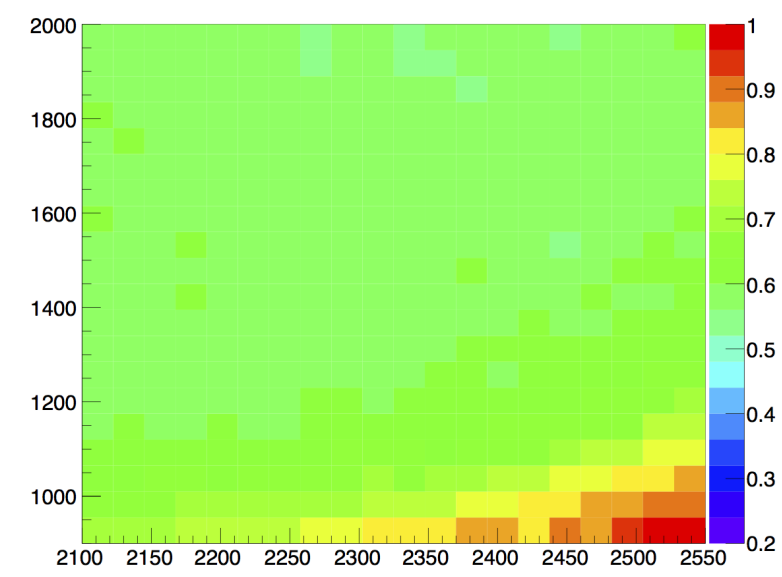

Figure 12. Map of the PSF size (sigma, in pixels) for a sensor segment.
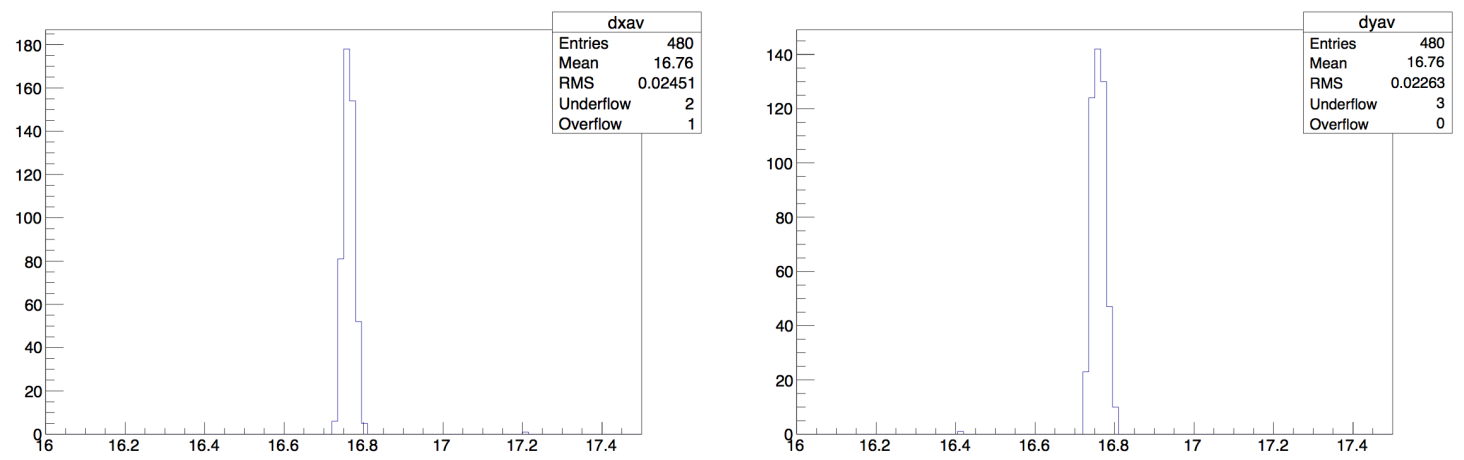

Figure 13. Distribution of distances between the neighboring holes along the row (left graph) and column (right graph) directions [in pixels].

with RMS smaller than 0.025 pixels or $0.25 \mu \mathrm{m}$. The reconstructed pitch is equal to 16.76 pixels or $167.6 \mu \mathrm{m}$ and is the same in both directions. 
Finally, figure 14 shows the distance between neighboring pinholes (in pixels) as a function of the row number for a sensor segment. The distances were averaged over all pinholes corresponding to a particular column with error bars corresponding to RMS of the distribution. The distance can be viewed a "standard stick" to probe local astrometric and shape distortions due to the sensor effects and the graph shows good uniformity and precision compatible with $0.25 \mu \mathrm{m}$ RMS demonstrated in figure 13.

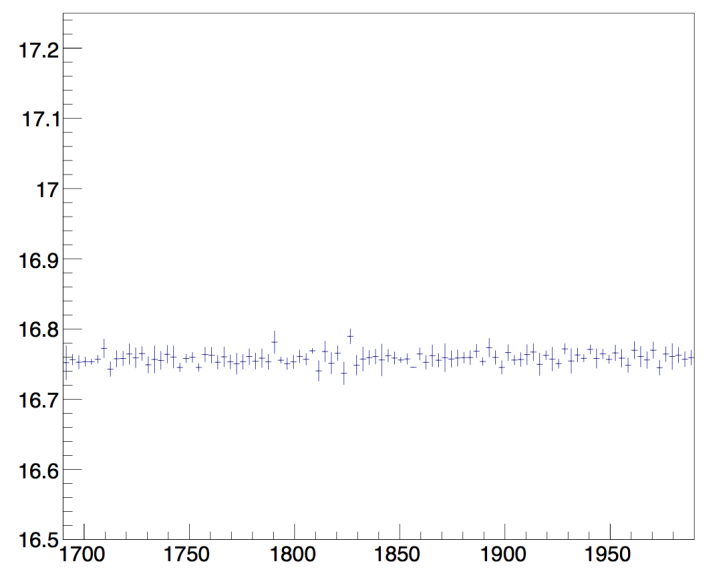

Figure 14. Distance between pinholes (in pixels) as a function of the row number for a sensor segment.

Further analysis showed that there is a longer-range (100's of pixels) variation of the measured distance of the order of $1 \mu \mathrm{m}$. Most likely this variation is caused by the optics because an independent metrology of the pinhole plate using OGP [9] confirmed regularity of the grid to be better than $0.5 \mu \mathrm{m}$. We therefore can conclude that this technique has potential to achieve submicron sensitivity and is a good candidate to study astrometric and shape biases in fully depleted CCD sensors. Work is in progress to apply this technique to the edge roll-off, midline and tree ring effects, such as reported in [10]. We also note that a similar approach was presented in another contribution to the workshop [11].

\section{Conclusions}

Achieving the precision dictated by the LSST science specifications will not be an easy task and the understanding of astrometry and photometry due to the CCD sensors will be one of the most important parts of this endeavor. In this manuscript we described the first results for two new techniques to probe astrometric and shape distortions due to the sensor: $\mathrm{x}$-ray flat fielding and emulating star fields by illuminating through arrays of pinholes. The $\mathrm{x}$-ray flat fielding is not sensitive to the surface so could help to disentangle photometric and astrometric (or pixel size) effects in the sensor. Regular grids of pinholes offer a simple way to study the distortions over the whole sensor area. Both techniques have been validated using known sensor anomalies and could be especially important for weak lensing applications. 


\section{Acknowledgments}

The author would like to thank Merlin Fisher-Levine and Paul Price for examples of the DM code; Erin Sheldon for help with the shape fitter; Paul O'Connor and Ivan Kotov for the datasets; John Warren for providing the plate with pinholes and Peter Takacs for the OGP measurements.

\section{References}

[1] S.M. Kahn et al., Design and development of the 3.2 gigapixel camera for the Large Synoptic Survey Telescope, Proc. SPIE 7735 (2010) 77350J.

[2] J.R. Janesick, Scientific Charge-Coupled Devices, SPIE Press, Bellingham, Washington U.S.A. (2001).

[3] I.V. Kotov, J. Frank, A.I. Kotov, P. Kubanek, P. O'Connor et al., Charge diffusion measurement in fully depleted CCD using X-rays, Nucl. Instrum. Meth. A 695 (2012) 296.

[4] T. Axelrod, J. Kantor, R.H. Lupton and F. Pierfederici, An open source application framework for astronomical imaging pipelines, Proc. SPIE 7740 (2010) 774015.

[5] E. Sheldon, An implementation of Bayesian Lensing Shear Measurement, Mon. Not. R. Astron. Soc. 444 (2014) L25 [arXiv: 1403.7669].

[6] P. O'Connor, Spot scan probe of lateral field effects in a thick fully depleted CCD, 2014 JINST 9 C03033.

[7] J. Warren, private communication. The plate was manufactured using facilities of the BNL Instrumentation Division.

[8] SAO Image DS9 Reference Manual http://ds9.si.edu/doc/ref/.

[9] P. Takacs, BNL Instrumentation Division, private communication. The measurement used the OGP Smartscope Quest 300.

[10] A.A. Plazas, G.M. Bernstein and E.S. Sheldon, Transverse electric fields' effects in the Dark Energy Camera CCDs, 2014 JINST 9 C04001 [arXiv: 1403.6127].

[11] A. Bradshaw, C. Lage, E. Resseguie, and J.A. Tyson, Mapping charge transport effects in thick CCDs with a dithered array of 40,000 stars, 2015 JINST 10 C04034. 